\title{
Design of simulation models and study on their basis the dynamics of the cyclic mechanisms
}

\author{
Viktor Telegin ${ }^{1, *}$ and Igor Telegin ${ }^{1}$ \\ ${ }^{1}$ Lipetsk State Technical University, Lipetsk, Russia
}

\begin{abstract}
Reliability and performance of mechanical systems of cyclic action are largely determined by the level of oscillatory processes in their links. Violation accuracy positioning of the output links mechanisms, the destruction of their elements during operation, accelerated wear - all this is the result of the presence of dynamic forces in the mechanism, the speed of the elements of which cyclically changes from a certain minimum to maximum value. Calculations of such mechanisms are based on their dynamic models. A model is a representation of a mechanism in the form of a system of concentrated masses connected by elastic-dissipative, piecewise-continuous (due to the presence of gaps), holonomic bonds. This work is devoted to automation processes for creating such models and calculating on their basis the dynamic characteristics of the mechanism.
\end{abstract}

\section{Simulation model of the mechanism}

In the context of this work, by simulation we mean a method in which the real mechanism, with a fairly high level of accuracy, is replaced by a lumped mass system, interconnected by kinematic, elastic-dissipative, in the general case, non-linear and breakable links. Such systems are called dynamic models, and their mathematical description is called a mathematical model. An experimental study on the basis of such models (numerical experiment) makes it possible to draw conclusions about the degree of reliability, operability of the mechanism for given modes of its operation, as well as determine the optimal parameters of these modes [1, 2, 3].

\section{The mathematical model of the mechanism}

The motion of a finite mass system on which ideal, stationary, holonomic constraints are superimposed can be described by systems of nonlinear differential equations of the second order - a mathematical model. To compose such systems, a special form of the secondorder Lagrange equation with redundant coordinates is often used [4].

However, this approach is unsuitable if you want to automate the process of developing a mathematical model of the system, obtained by joining additional links to it $[5,6]$. Figure 1 shows the process of creating a mathematical model of a cyclic mechanism, in which, based

* Corresponding author: vv.telegin@yandex.ru 
on the methods of component modeling, the simulation model itself and its mathematical description are built.
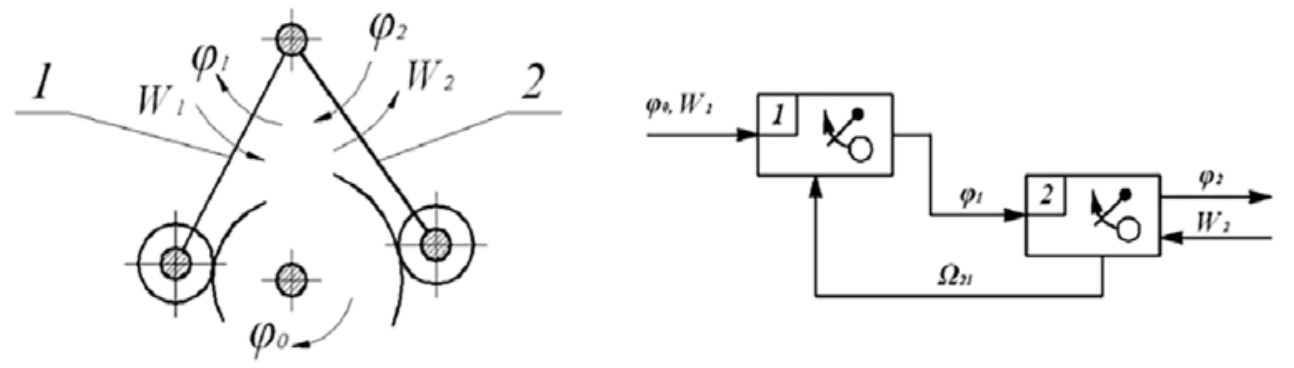

Fig. 1. Cam mechanism and its object model.

$$
\left\{\begin{array}{l}
J_{1} \ddot{\varphi}_{1}=-c_{1} \cdot\left(\varphi_{1}-U_{1}\right)-b_{1} \cdot\left(\dot{\varphi}_{1}-\dot{U}_{1}\right)+W_{1}+\Omega_{21} \\
J_{2} \ddot{\varphi}_{2}=-c_{3} \cdot\left(\varphi_{2}-\varphi_{1}\right)-b 3 \cdot\left(\dot{\varphi}_{2}-\dot{\varphi}_{1}\right)+W 2
\end{array},\right.
$$

where external forces are friction and locking force:

$$
\begin{aligned}
& W_{1}=-M_{f 1}-M_{c l}, \\
& W 2=-M_{f 2}-M c l-c_{2} \cdot\left(\varphi_{2}-U_{2}\right)-b_{2} \cdot\left(\dot{\varphi}_{2}-\dot{U}_{2}\right) .
\end{aligned}
$$

The impact of the next object on the current object (response):

$$
\Omega_{21}=c_{3} \cdot\left(\varphi_{2}-\varphi_{1}\right)+b_{3} \cdot\left(\dot{\varphi}_{2}-\dot{\varphi}_{1}\right),
$$
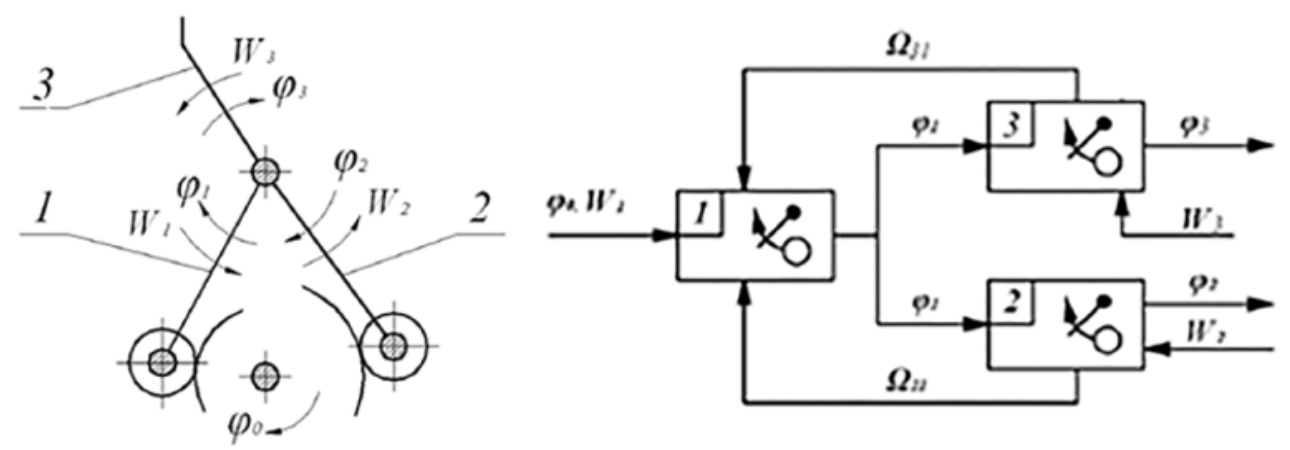

Fig. 2. Joining an additional lever to the cam mechanism.

$$
J_{3} \ddot{\varphi}_{3}=-c_{3} \cdot\left(\varphi_{3}-\varphi_{1}\right)-b_{3} \cdot\left(\dot{\varphi}_{3}-\dot{\varphi}_{1}\right)+W_{3}
$$

where $W_{3}=-M_{f r}, a \Omega_{31}=\left(c_{3}\left(\varphi_{3}-U_{3}\right)+\left(\dot{\varphi}_{3}-\dot{U}_{3}\right)\right) U_{3}^{\prime}$.

Equation (4) is one more equation to system (1). 

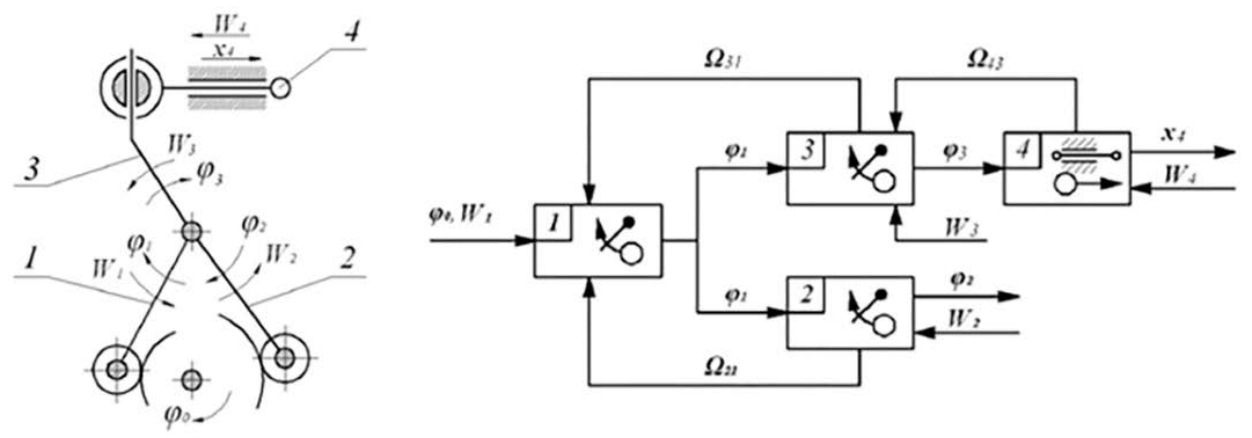

Fig. 3. Joining a translationally motioning link to the cam-and-lever mechanism.

The kinematic diagram and object model shown in Figure 3 completes the formation of a component model of the cutting mechanism cold-stamping automatic machine (CSAM) $A B 1818$ and, importantly, its mathematical model (5).

$$
\left\{\begin{array}{l}
J_{1} \ddot{\varphi}_{1}=-c_{1} \cdot\left(\varphi_{1}-U_{1}\right)-b_{1} \cdot\left(\dot{\varphi}_{1}-\dot{U}_{1}\right)+W_{1}+\Omega_{21} \\
J_{2} \ddot{\varphi}_{2}=-c_{3} \cdot\left(\varphi_{2}-\varphi_{1}\right)-b 3 \cdot\left(\dot{\varphi}_{2}-\dot{\varphi}_{1}\right)+W 2 \\
J_{3} \ddot{\varphi}_{3}=-c_{3} \cdot\left(\varphi_{3}-\varphi_{1}\right)-b_{3} \cdot\left(\dot{\varphi}_{3}-\dot{\varphi}_{1}\right)+W_{3} \\
m_{4} \ddot{x}_{4}=-c_{4}\left(x_{4}-U_{4}\right)-b_{4}\left(\dot{x}_{4}-\dot{U}_{4}\right)+W_{4}
\end{array}\right.
$$

In equations (5): $W_{4}=-P_{f r}, a \Omega_{43}=\left(c_{4}\left(x_{4}-U_{4}\right)+\left(\dot{x}_{4}-\dot{U}_{4}\right)\right) U_{4}^{\prime}$.

\section{Software implementation of the simulation model}

Figure 4 shows the 3D model [7] of cutting mechanism of the five-position, nuts cold stamping machine. Using it as example, a methodology was developed for creating a mathematical model (5) in an interactive mode.

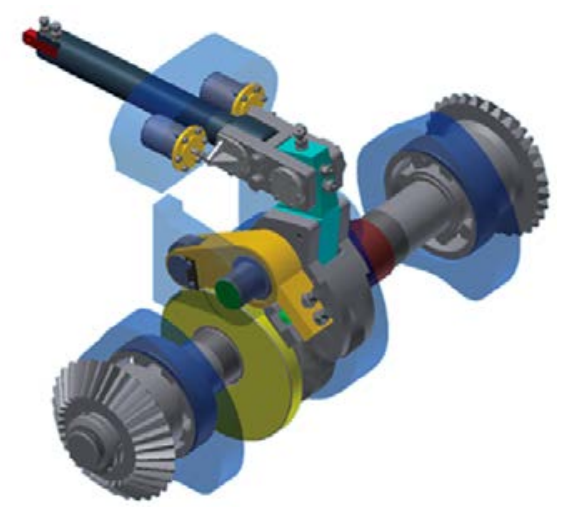

Fig. 4. 3D model of the cutting mechanism of the cold-stamping machine.

Each of the objects of this model (Figure 5) is typical. For each of the objects, several variants of dynamic models have been developed, differing in the number of lumped masses (model complexity), the presence and types of gaps in kinematic pairs, methods for taking into account friction forces and energy dissipation, as well as other parameters. The user has the opportunity to evaluate the operability of each of the objects of the mechanism 
under various operating conditions, and as a result, to assemble a system that meets the requirements for performing simulation operations.

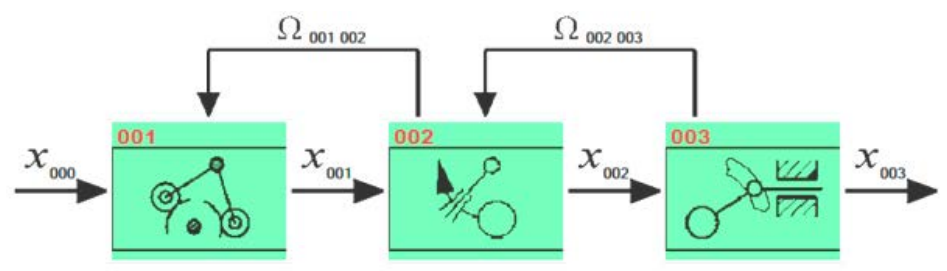

Fig. 5. The object model of the cutting mechanism CSAM AB1818.

"Dam" software interface uses other notation than in expressions (1) - (5). All movements of the input and output links of objects are indicated by the symbol " $x$ " with indices of the input and output links differing by one. The response has two characters denoting the previous and next objects.

\section{Evaluation of the reliability and operability of the cutting mechanism CSAM AB1818 based on its simulation model}

The paper presents the results of a study in the software "dam" [8] dynamic processes that affect the positioning accuracy of the executive link of cutting mechanism of the fiveposition CSAM AB1818 for stamping nuts. This device not only cuts the workpiece from the bar, but also transfers it to the first stamping position. The model of studied mechanism (Figure 5) includes three objects: the cam mechanism, the upper arm of the forward lever and the knife stem. The initial data for performing dynamic calculations are the elastic inertia properties of the elements of the investigated product, forces (dissipation, friction, structural and technological), gaps in kinematic pairs, accuracy parameters for manufacturing higher pairs (cams).

Tasks of studying the dynamics of the cutting mechanism:

- dynamic analysis of the mechanism depending on the speed of its operation and the degree of the kinematic pairs wear;

- evaluation the influence of cams profiling laws on dynamic processes occurring in a mechanism;

- impact analysis of the manufacturing accuracy of cam tracks on the mechanism dynamics.

As criteria for the reliability and operability of the cutting mechanism, will use the maximum deviations of the movement and speed for the executive link from ideal. Figure 6 shows the calculated values of the maximum deviation of the displacement and speed of the executive link of the cutting mechanism when the force of preliminary tightening of the springs is $500 \mathrm{~N}$ and the gap is $0.25 \mathrm{~mm}$.

An analysis of the dependencies in Figure 6 shows that at speeds of the mechanism up to 300 strokes per minute, the value of positioning accuracy does not differ significantly depending on the speed of the mechanism. An increase the gaps in the kinematic pairs within the studied limits leads to growth in the observed parameters.

The explanation for this is obvious. The peak in the amplitude of the oscillatory processes that occur after the technological load is dumped falls precisely on the section of the upper dwell. The executive link is accelerated to maximum speed immediately after the technological effort is discharged even in the forward stroke section. Reliable operation of the mechanism can be guaranteed at speeds not exceeding 300 strokes per minute, which coincides with the data of the manufacturer. 

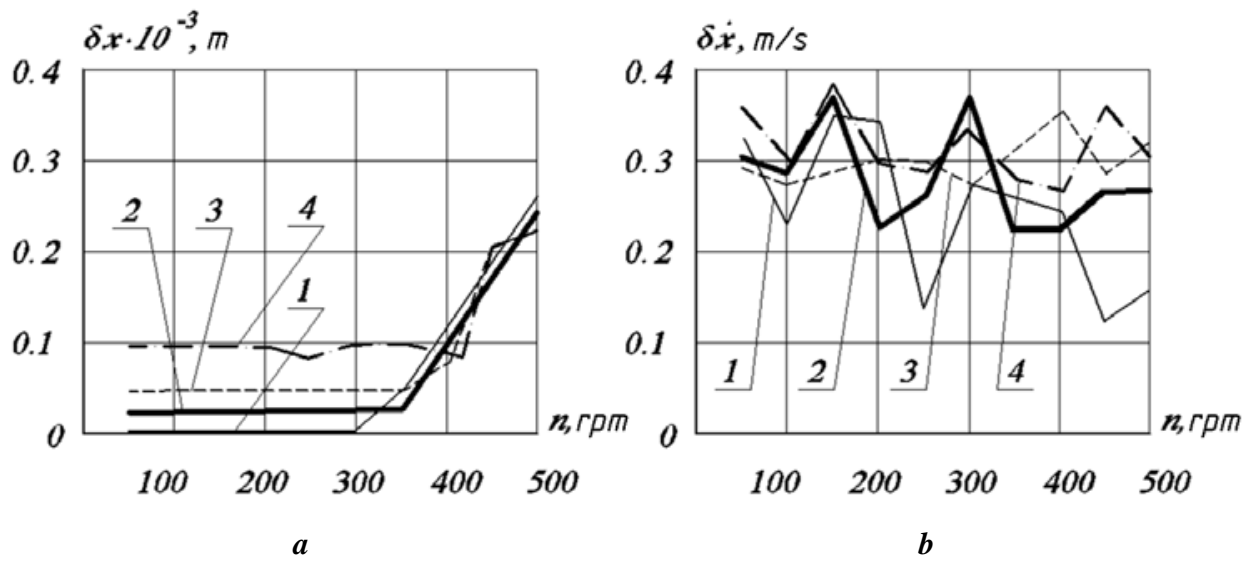

Fig. 6. The maximum deviations of the movement $(\boldsymbol{a})$ and speed $(\boldsymbol{b})$ of the executive link of the cutting mechanism $C S A M A B 1818$ with the size of the gaps in the kinematic pairs: $1-0 \mu m$, $2-25 \mu \mathrm{m}, 3-50 \mu \mathrm{m}, \mathbf{4}-75 \mu \mathrm{m}$.

In carrying out further research, we will assume the tightening force is $500 \mathrm{~N}$ and the gap is $0.25 \mathrm{~mm}$, and the calculation of the criteria should be carried out for the full cycle of the mechanism.

Data on the dynamics of the cutting mechanism with cams profiled according to different laws are shown in the Table 1 (positioning accuracy $-\delta x_{\max }, \mu m$; maximum speed $\left.-\dot{x}_{\max }, m / s\right)$.

Table 1. The results of dynamic studies of the cutting mechanism CSAM AB1818.

\begin{tabular}{|c|c|c|c|c|c|c|}
\hline \multirow{2}{*}{ Cam track profile } & \multicolumn{6}{|c|}{ Kinematic gaps $25 / 50, \mu \mathrm{m}$} \\
\cline { 2 - 7 } & \multicolumn{2}{|c|}{ Speed 110} & \multicolumn{2}{c|}{ Speed 260 } & \multicolumn{2}{c|}{ Speed 500 } \\
\cline { 2 - 7 } & $\delta x_{\max }$ & $\dot{x}_{\max }$ & $\delta x_{\max }$ & $\dot{x}_{\max }$ & $\delta x_{\max }$ & $\dot{x}_{\max }$ \\
\hline \multirow{2}{*}{$\begin{array}{c}\text { Uniformly } \\
\text { accelerated }\end{array}$} & $\frac{83.5}{106.9}$ & $\frac{0.46}{0.40}$ & $\frac{85.1}{109.2}$ & $\frac{0.87}{0.89}$ & $\frac{233.0}{239.3}$ & $\frac{1.56}{1.75}$ \\
\hline \multirow{2}{*}{ Sinusoidal } & $\frac{83.6}{106.8}$ & $\frac{0.48}{0.53}$ & $\frac{83.2}{107.5}$ & $\frac{0.92}{0.95}$ & $\frac{264.0}{279.4}$ & $\frac{1.69}{1.74}$ \\
\hline \multirow{2}{*}{ Cosinusoidal } & $\frac{83.6}{107.4}$ & $\frac{0.33}{0.37}$ & $\frac{82.5}{110.8}$ & $\frac{0.72}{0.75}$ & $\frac{167.5}{171.9}$ & $\frac{1.37}{1.43}$ \\
\hline
\end{tabular}

As follows from the data in the table, one cannot speak of a pronounced dependence of the intensity of dynamic processes in the cutting mechanism on the form of the law of profiling cam tracks. At the same time, such a dependence exists. Accordingly, the statement of the problem of searching for the law of profiling of cam tracks, providing the best dynamic characteristics of the mechanism, is quite advisable.

To assess the impact of manufacturing accuracy of cams on the dynamics of the cutting mechanism, we use the technique developed by the author [8]. In this case, we assume that the wear of the kinematic pairs of the mechanism is minimal; the maximum profile error $\chi_{\max }$ is $40 \mu \mathrm{m}$. The calculated data correspond to the cases of profiling the cams of the cutting mechanism according to the sinusoidal (Figure 7) and cosinusoidal (Figure 8) laws of change in acceleration. 

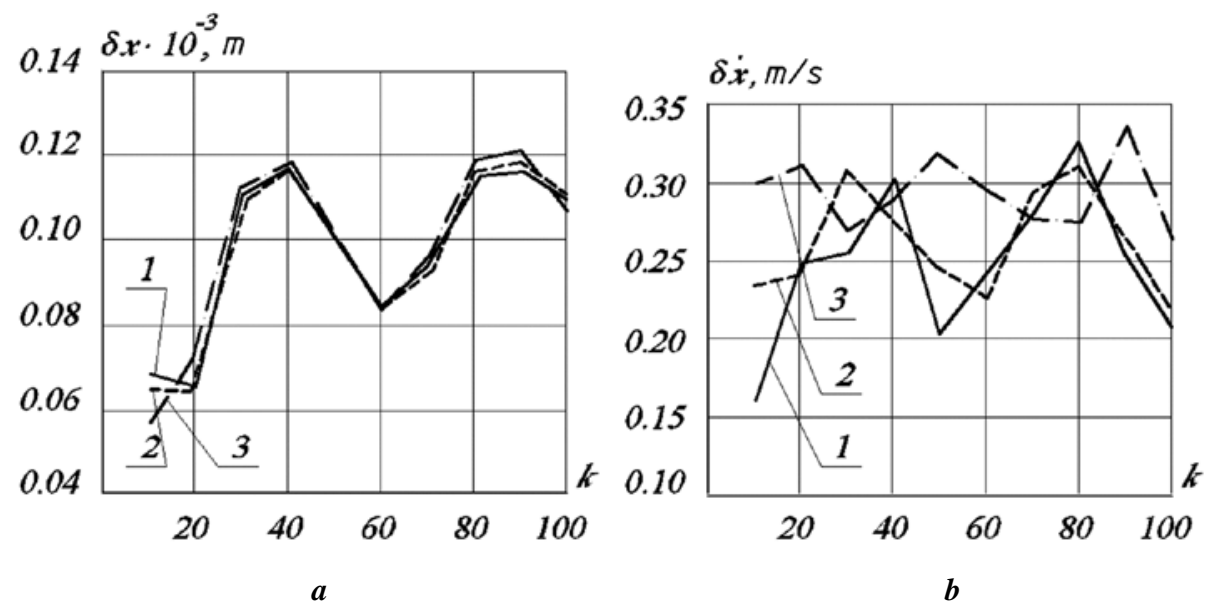

Fig. 7. Deviations from the ideal movement $(\boldsymbol{a})$ and speed $(\boldsymbol{b})$ of the executive link of the cutting mechanism CSAM AB1818; the law of profiling of cam tracks is sinusoidal; work speed: $1-110$, $2-180,3-260$ strokes per minute.
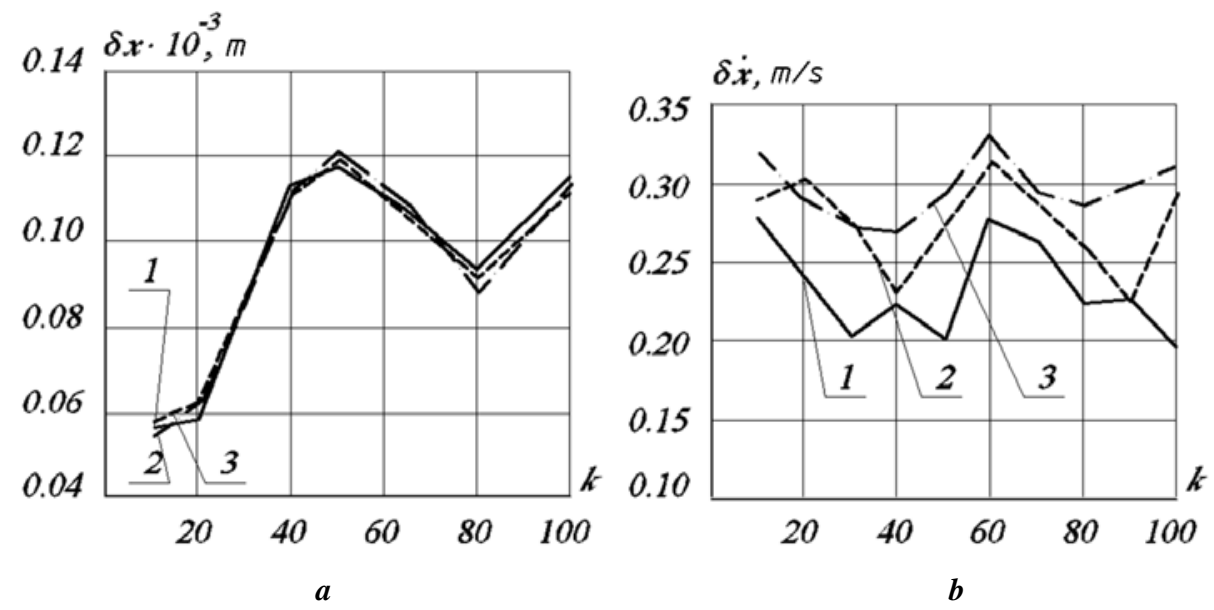

Fig. 8. Deviations from the ideal movement $(\boldsymbol{a})$ and speed $(\boldsymbol{b})$ of the executive link of the cutting mechanism CSAM AB1818; the law of profiling of cam tracks is cosinusoidal; work speed: $1-110$, 2 - 180, 3 - 260 strokes per minute.

\section{Conclusions}

From the analysis of the given dependences, it follows that the intensity of the dynamic processes in the cutting mechanism reaches a maximum with a coefficient $\mathrm{k}$ characterizing the cam track profile error in the range from 20 to 80, and at all speed modes recommended for the operation of the machine. The study of the dynamics of the segment mechanism taking into account the error in the manufacture of cam tracks allows us to make the assumption that the relationship between the level of dynamic processes in the mechanism, the type of cam profiling law and the error in their manufacture is most likely insignificant.

This research was financially supported by the Ministry of Science and Education of Russia (project No. 11.9505.2017/8.9). 


\section{References}

1. A.M. Law, W.D. Kelton, Imitacionnoe modelirovanie (SPb.: Peter, 2004)

2. J. Rumbaugh et al., Object-Oriented Modeling and Design (Prentice Hall, 1991)

3. I.A. Krivosheev, D.A. Ahmedzyanov, Modelirovanie dinamicheskih processov v slozhnyh sistemah (UFA.: UGATU, 2003)

4. I.I. Wulfson, Vibrations in machines with cyclic action mechanisms (Leningrad: Mashinostroine, 1990)

5. E.S. Ben'kovich, YU.B. Kolesov, YU.B. Senichenkov, Prakticheskoe modelirovanie dinamicheskih system (SPb.: BHV, 2002)

6. V. Telegin, A. Kozlov, T. Shumilova, Development and research of the rotating lever object as a dynamic model of a cycle mechanism, MATEC Web Conf. International Conference on Modern Trends in Manufacturing Technologies and Equipment (ICMTMTE 2018), v. 224, pp. 02078 (2018)

7. V.V. Telegin, A.M. Kozlov, A.V. Kirichek, Solid Modeling in Autodesk Inventor at Initial Stage of Training of Specialists in Field Mechanical Engineering, Lecture Notes In Mechanical Engineering ISSN: 2195-4356, v. Part F4, pp. 1241-1247 (2019)

8. V. Telegin, A. Kozlov, Computer realization of research into the dynamics of mechanical systems, IOP Conference Series: Materials Science and Engineering, v. 124, pp. 012101 (2016) 